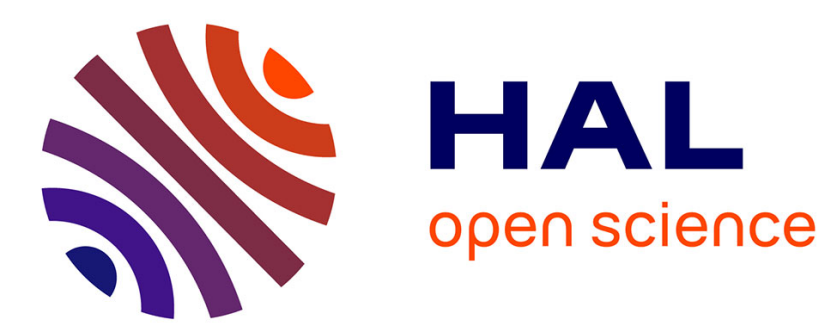

\title{
THE DISCRETE LAPLACIAN ACTING ON 2-FORMS AND APPLICATION
}

Hatem Baloudi, Sayda Belgacem, Aref Jeribi

\section{To cite this version:}

Hatem Baloudi, Sayda Belgacem, Aref Jeribi. THE DISCRETE LAPLACIAN ACTING ON 2FORMS AND APPLICATION. Bulletin of the Malaysian Mathematical Sciences Society, In press. hal-01758938v2

\section{HAL Id: hal-01758938 \\ https://hal.science/hal-01758938v2}

Submitted on 7 Oct 2018

HAL is a multi-disciplinary open access archive for the deposit and dissemination of scientific research documents, whether they are published or not. The documents may come from teaching and research institutions in France or abroad, or from public or private research centers.
L'archive ouverte pluridisciplinaire HAL, est destinée au dépôt et à la diffusion de documents scientifiques de niveau recherche, publiés ou non, émanant des établissements d'enseignement et de recherche français ou étrangers, des laboratoires publics ou privés. 


\title{
THE DISCRETE LAPLACIAN ACTING ON 2-FORMS AND APPLICATION
}

\author{
HATEM BALOUDI, SAYDA BELGACEM, AND AREF JERIBI
}

\begin{abstract}
In the current paper, we study the discrete Laplacian acting on 2-forms which was introduced and investigated by Chebbi (2018). We establish a new criterion of essential self-adjointness using the Nelson lemma. Moreover, we give an upper bound on the infimum of the essential spectrum. Furthermore, we establish a link between the adjacency matrix and the discrete Laplacian on 2-forms.
\end{abstract}

\section{Contents}

1. Introduction

2. Generalities about graphs

3. The symmetric and skew-symmetric spaces 4

3.1. Hilbert structures on the set of edges 4

3.2. Hilbert structures on the set of faces 5

4. Operators 5

4.1. Skew-symmetric case 6

4.2. Symmetric case 6

4.3. Relationship between $\mathcal{L}_{2, \text { skew }}$ and $\mathcal{L}_{2, \text { sym }} \quad 7$

5. A Nelson criterium 8

6. Essential spectrum 11

7. Application to the study of the adjacency matrix 13

$\begin{array}{ll}\text { 7.1. Adjacency matrix } & 13\end{array}$

7.2. Triangular graph 13

$\begin{array}{ll}\text { 7.3. } & \text { Geometric Hypothesis } \\ \end{array}$

$\begin{array}{ll}\text { 7.4. Book-like triangulation } & 16\end{array}$

$\begin{array}{ll}\text { 7.5. Triangular anti-tree } & 17\end{array}$

$\begin{array}{ll}\text { References } & 18\end{array}$

\section{INTRODUCTION}

Spectral graph theory represents an active area of research. In the last few years, the questions of the essential self-adjointness of discrete Laplacian

2010 Mathematics Subject Classification. 05C12, 05C63, 47B25, 81Q35.

Key words and phrases. locally finite graph, essential self-adjointness, adjacency matrix, unboundedness, spectral graph theory. 
operators on infinite graphs have attracted a lot of interest, see $[11,18,22$, 17]. There exist other definitions of the discrete Laplacian, e.g., [24, 16, 4, 2]. The one we are studying here is the discrete Laplacian acting on 2-forms and denoted by $\mathcal{L}_{2, \text { skew }}$, where skew stands for skew-symmetric. This operator was introduced by Chebbi in [7]. The author shows the relation between the $\chi$-completeness geometric hypothesis for the graph and essentially selfadjointness for the discrete Laplacian $\mathcal{L}_{2 \text {,skew }}$. More specifically, the author has proved that $\mathcal{L}_{2 \text {,skew }}$ is essential self-adjoint, when the triangulation (we refer to Section 2 for a precise definition) is $\chi$-complete.

The current study has two major aims. It first aims to discuss the question

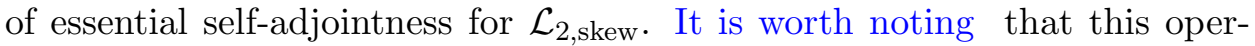
ator depends on the weight $\mathcal{R}$ on oriented triangular faces and the weight $\mathcal{E}$ on oriented edges, see Section 4 for more details. In the setting of electrical networks, the weight $\mathcal{E}$ correspond to the conductance. We establish a hypothesis on the weights and involve essential self-adjointness by using the Nelson commutator theorem. The technique of the proof are inspired from [3]. Moreover, we give an upper bound on the infimum of the essential spectrum $\sigma_{\text {ess }}\left(\mathcal{L}_{2 \text {,skew }}^{\mathcal{F}}\right)$, where $\mathcal{L}_{2 \text {,skew }}^{\mathcal{F}}$ is the Friedreichs extension of $\mathcal{L}_{2 \text {,skew }}$. Secondly, the paper aims to identify the link between the adjacency matrix and the discrete Laplacian $\mathcal{L}_{2 \text {,skew }}$. To achieve this goal, we analyze the structure of $\mathcal{L}_{2, \text { skew }}$. Note that this discrete Laplacian was introduced on a skew-symmetric statistic on the space of 2 -forms. We can define the discrete Laplacian $\mathcal{L}_{2, \text { sym }}$ in the symmetric case by the same expression of $\mathcal{L}_{2, \text { sym. }}$. In the case of a tri-partite graph, we prove that the two operators are unitarily equivalent. Furthermore, $\mathcal{L}_{2 \text {,sym }}$ is unitarily equivalent to the adjacency matrix of the triangular graph, see Section 7 for more details. We recall that the spectral theory of adjacency matrix acting on graphs is useful for the study of some gelling polymers, of some electrical networks, and in number theory, see $[14,13,23]$.

As for the rest of this paper, it is structured as follows: The next section is devoted to some definitions and notations for graph. We find the definitions of two different Hilbert structures on the set of faces, in Section 3. Both definitions have their own interest. This permits to define two different types of discrete Laplacian associated to faces. The relation between these two operators is clearly presented in Section 4.3. In Section 5, we discuss the question of essential self-adjointness for the discrete Laplacian $\mathcal{L}_{2 \text {,skew }}$. We establish a new criterion of essential self-adjointness using the Nelson lemma. In Section 6, we give an upper bound on the infimum of the essential spectrum. The obtained findings from the previous sections are presented in Section 7 for the purpose of investigating the questions of boundedness and essential self-adjointness for the adjacency matrix.

Acknowledgment. The authors thank Colette Anné, Nabila TorkiHamza, Sylvain Golénia and Nassim Athmouni for useful discussions and 
comments on the text. They would like to thank also the anonymous referee for their numerous relevant remarks and useful suggestions.

\section{Generalities about GRAphs}

We start with some definitions to fix notations for graphs and refer to $[9,10,24]$ for surveys on the matter. Let $\mathcal{V}$ be a countable set. We equip $\mathcal{V}$ with the discrete topology. Let $\mathcal{E}: \mathcal{V} \times \mathcal{V} \longrightarrow[0,+\infty)$ and assume that $\mathcal{E}$ is symmetric (i.e., $\mathcal{E}(x, y)=\mathcal{E}(y, x)$, for all $x, y \in \mathcal{V})$. Let $m: \mathcal{V} \longrightarrow(0,+\infty)$. We say that $\mathcal{G}=(\mathcal{V}, m, \mathcal{E})$ is a weighted graph with vertices $\mathcal{V}$, weight of vertices $m$ and weight of edges $\mathcal{E}$. In the setting of electrical networks, the weights correspond to the conductances. We say that $x, y$ are neighbors if $\mathcal{E}(x, y) \neq 0$ and we denote it by $x \sim y$. A graph $\mathcal{G}$ is simple if it has no loops (i.e., $\mathcal{E}(x, x)=0), \mathrm{m}=1$ and $\mathcal{E}$ has values in $\{0,1\}$. The set of neighbors of $x \in \mathcal{V}$ is denoted by

$$
\mathcal{N}_{\mathcal{G}}(x):=\{y \in \mathcal{V}: \mathcal{E}(x, y) \neq 0\} .
$$

A graph is locally finite if $\sharp \mathcal{N}_{\mathcal{G}}(x)$ is finite for all $x \in \mathcal{V}$. The weighted degree of vertices is given by

$$
d_{\mathcal{V}}(x):=\frac{1}{m(x)} \sum_{y \sim x} \mathcal{E}(x, y) .
$$

When $\mathcal{G}$ is simple, $d_{\mathcal{V}}(x)=\sharp \mathcal{N}_{\mathcal{G}}(x)$. A graph $\mathcal{G}$ is connected, if for all $x, y \in$ $\mathcal{V}$, there exists an $x-y$-path, i.e., there is a sequence $\left(x_{1}, \ldots, x_{N+1}\right) \in \mathcal{V}^{N+1}$ such that $x_{1}=x, \quad x_{N+1}=y$ and $\mathcal{E}\left(x_{n}, x_{n+1}\right)>0$ for all $n \in\{1, \ldots, N\}$. If no vertices appear more than once in $\left(x_{1}, \ldots, x_{N}\right)$, the path $\left(x_{1}, \ldots, x_{N+1}\right)$ is called a simple path. The path is called a cycle or closed when the origin and the end are identical, i.e., $x_{1}=x_{N+1}$. An $n$-cycle is a cycle with $n$ vertices.

\section{In the sequel, we shall always consider graphs $\mathcal{G}$, which are locally finite, connected and have no loop.}

The set of cyclic permutations of $(x, y, z) \in \mathcal{V}^{3}$ is denoted by

$$
\circlearrowleft(x, y, z):=\{(x, y, z),(y, z, x),(z, x, y)\} .
$$

Let $\operatorname{Tr}$ the set of all simple 3-cycles

$$
\mathcal{F}=\operatorname{Tr} / \cong
$$

where $\varpi_{1} \cong \varpi_{2}$ if and only if $\varpi_{1}$ is a cyclic permutation of $\varpi_{2}$. The elements of $\mathcal{F}$ are called triangular faces. Atriangulation is a couple $(\mathcal{G}, \mathcal{F})$ where $\mathcal{G}$ is a graph and $\mathcal{F}$ is the set of all triangular faces.

In the sequel, we represent the triangular faces by their vertices. For a triangular face $\varpi=(x, y, z)$, we have

$$
\varpi=(x, y, z)=(y, z, x)=(z, x, y) .
$$


Let $(\mathcal{G}=(\mathcal{V}, \mathcal{E}, m), \mathcal{F})$ be a triangulation and let $\mathcal{R}: \mathcal{V} \times \mathcal{V} \times \mathcal{V} \longrightarrow[0,+\infty)$ such that

$$
(x, y, z) \in \mathcal{F} \Longleftrightarrow \mathcal{R}(x, y, z)>0 .
$$

We assume that $\mathcal{R}$ is symmetric, i.e.

$$
\mathcal{R}(x, y, z)=\mathcal{R}(\sigma(x, y, z))
$$

For all $(x, y, z) \in \mathcal{V} \times \mathcal{V} \times \mathcal{V}$ and for any permutation $\sigma(x, y, z)$ of $(x, y, z)$. We say that $\mathcal{T}=(\mathcal{V}, m, \mathcal{E}, \mathcal{R})$ is a weighted triangulation with weight of triangular faces $\mathcal{R}$. We say that $\mathcal{T}$ is simple if $\mathcal{G}:=(\mathcal{V}, m, \mathcal{E})$ is simple and the weights of the faces equal 1. Choosing an orientation of triangulation consists of defining a partition of $\mathcal{F}$ :

$$
\begin{gathered}
\mathcal{F}=\mathcal{F}^{+} \sqcup \mathcal{F}^{-}, \\
\left(x_{1}, x_{2}, x_{3}\right) \in \mathcal{F}^{+} \Longleftrightarrow\left(x_{3}, x_{2}, x_{1}\right) \in \mathcal{F}^{-} .
\end{gathered}
$$

For $\left(x_{1}, x_{2}, x_{3}\right) \in \mathcal{F}$, we denote

$$
-\left(x_{1}, x_{2}, x_{3}\right)=\left(x_{3}, x_{2}, x_{1}\right) .
$$

The set of neighbors of the edge $(x, y)$ is given by

$$
\mathcal{F}_{(x, y)}:=\mathcal{N}_{\mathcal{G}}(x) \cap \mathcal{N}_{\mathcal{G}}(y)
$$

The weighted degree of edges is given by:

$$
d_{\mathcal{E}}(x, y):=\frac{1}{\mathcal{E}(x, y)} \sum_{z \in \mathcal{F}_{(x, y)}} \mathcal{R}(x, y, z) .
$$

When $\mathcal{T}$ is simple, $d_{\mathcal{E}}(x, y)=\sharp \mathcal{F}_{(x, y)}$.

\section{The Symmetric AND SKeW-SyMmetric SPACES}

3.1. Hilbert structures on the set of edges. Let $\mathcal{T}=(\mathcal{V}, m, \mathcal{E}, \mathcal{R})$ be a weighted triangulation. Let $\mathcal{E}:=\{(x, y) \in \mathcal{V} \times \mathcal{V}: \mathcal{E}(x, y)>0\}$. The set of 1 -cochains (or 1-forms) is given by:

$$
\mathcal{C}_{\text {skew }}(\mathcal{E}):=\{f: \mathcal{E} \rightarrow \mathbb{C}, f(x, y)=-f(y, x) \text { for all } x, y \in \mathcal{V}\},
$$

where skew stands for skew-symmetric. This corresponds to fermionic statistics. The set of functions with finite support is denoted by $\mathcal{C}_{\text {skew }}^{c}(\mathcal{E})$. Concerning bosonic statistics, we define:

$$
\mathcal{C}_{\text {sym }}(\mathcal{E}):=\{f: \mathcal{E} \rightarrow \mathbb{C}, f(x, y)=f(y, x) \text { for all } x, y \in \mathcal{V}\} .
$$

The set of functions with finite support is denoted by $\mathcal{C}_{\text {sym }}^{c}(\mathcal{E})$.

We turn to the Hilbert structures.

$\ell_{\text {skew }}^{2}(\mathcal{E}):=\left\{f \in \mathcal{C}_{\text {skew }}(\mathcal{E})\right.$ such that $\left.\|f\|^{2}:=\frac{1}{2} \sum_{x, y \in \mathcal{V}} \mathcal{E}(x, y)|f(x, y)|^{2}<\infty\right\}$ 
and

$\ell_{\mathrm{sym}}^{2}(\mathcal{E}):=\left\{f \in \mathcal{C}_{\mathrm{sym}}(\mathcal{E})\right.$ such that $\left.\|f\|^{2}:=\frac{1}{2} \sum_{x, y \in \mathcal{V}} \mathcal{E}(x, y)|f(x, y)|^{2}<\infty\right\}$.

The associated scalar product is given by

$$
\langle f, g\rangle:=\frac{1}{2} \sum_{(x, y) \in \mathcal{E}} \mathcal{E}(x, y) \overline{f(x, y)} g(x, y),
$$

when $f$ and $g$ are both in $\ell_{\text {skew }}^{2}(\mathcal{E})$ or in $\ell_{\text {sym }}^{2}(\mathcal{E})$.

3.2. Hilbert structures on the set of faces. Let $\mathcal{T}=(\mathcal{V}, m, \mathcal{E}, \mathcal{R})$ be a weighted triangulation. The set of 2 -cochains or 2 -forms is given by

$$
\mathcal{C}_{\text {skew }}(\mathcal{R})=\{f: \mathcal{F} \longrightarrow \mathbb{C}: f(x, y, z)=-f(z, y, x)\} .
$$

The set of functions with finite support is denoted by $\mathcal{C}_{\text {skew }}^{c}(\mathcal{R})$. Concerning the case symmetric, we define

$$
\mathcal{C}_{\text {sym }}(\mathcal{R})=\{f: \mathcal{F} \longrightarrow \mathbb{C}: f(x, y, z)=f(z, y, x)\} .
$$

The set of functions with finite support is denoted by $\mathcal{C}_{\text {sym }}^{c}(\mathcal{R})$. Let us define the Hilbert spaces $\ell_{\text {skew }}^{2}(\mathcal{R})$ and $\ell_{\text {sym }}^{2}(\mathcal{R})$ as the sets of cochains with finite norm, we have

$$
\ell_{\text {skew }}^{2}(\mathcal{R}):=\left\{f \in \mathcal{C}_{\text {skew }}(\mathcal{R}):\|f\|^{2}=\frac{1}{2} \sum_{(x, y, z) \in \mathcal{F}} \mathcal{R}(x, y, z)|f(x, y, z)|^{2}<\infty\right\}
$$

and

$$
\ell_{\mathrm{sym}}^{2}(\mathcal{R}):=\left\{f \in \mathcal{C}_{\mathrm{sym}}(\mathcal{R}):\|f\|^{2}=\frac{1}{2} \sum_{(x, y, z) \in \mathcal{F}} \mathcal{R}(x, y, z)|f(x, y, z)|^{2}<\infty\right\} .
$$

The associated scalar product is given by

$$
\begin{aligned}
\langle f, g\rangle & :=\frac{1}{2} \sum_{(x, y, z) \in \mathcal{F}} \mathcal{R}(x, y, z) \overline{f(x, y, z)} g(x, y, z) \\
& :=\frac{1}{6} \sum_{(x, y) \in \mathcal{E}} \sum_{z \in \mathcal{F}_{(x, y)}} \mathcal{R}(x, y, z) \overline{f(x, y, z)} g(x, y, z)
\end{aligned}
$$

when $f$ and $g$ are both in $\ell_{\text {skew }}^{2}(\mathcal{R})$ or in $\ell_{\text {sym }}^{2}(\mathcal{R})$.

\section{Operators}

In this section, we recall the concept of exterior derivative operator associated to a faces space, we refer to $[7,8]$ for more details. This permits to define the discrete Laplacian acting on 2 -forms. 
4.1. Skew-symmetric case. We start with defining the operators in the skew-symmetric case. The skew-symmetric exterior operator is the operator $d_{\text {skew }}^{1}: \mathcal{C}_{\text {skew }}^{c}(\mathcal{E}) \longrightarrow \mathcal{C}_{\text {skew }}^{c}(\mathcal{R})$, given by

$$
d_{\text {skew }}^{1}(f)(x, y, z)=f(x, y)+f(y, z)+f(z, x) .
$$

The skew-symmetric co-exterior derivative operator is the formal adjoint of $d_{\text {skew }}^{1}$, i.e. it is the operator $\delta_{\text {skew }}^{1}: \mathcal{C}_{\text {skew }}^{c}(\mathcal{R}) \longrightarrow \mathcal{C}_{\text {skew }}^{c}(\mathcal{E})$, given by

$$
\forall f \in \mathcal{C}_{\text {skew }}^{c}(\mathcal{R}), \quad \delta_{\text {skew }}^{1}(f)(x, y)=\frac{1}{\mathcal{E}(x, y)} \sum_{z \in \mathcal{F}_{(x, y)}} \mathcal{R}(x, y, z) f(x, y, z)
$$

Both operators are closable (see [7, Lemme 3.1]). We denote their closure by the same symbol. The skew-symmetric discrete Laplacian operator acting on 2 -forms is given by

$$
\begin{aligned}
\mathcal{L}_{2, \text { skew }}(f)(x, y, z) & =d_{\text {skew }}^{1} \delta_{\text {skew }}^{1}(f)(x, y, z) \\
& =\frac{1}{\mathcal{E}(x, y)} \sum_{t \in \mathcal{F}_{(x, y)}} \mathcal{R}(x, y, t) f(x, y, t) \\
& +\frac{1}{\mathcal{E}(y, z)} \sum_{t \in \mathcal{F}_{(y, z)}} \mathcal{R}(y, z, t) f(y, z, t) \\
& +\frac{1}{\mathcal{E}(z, x)} \sum_{t \in \mathcal{F}_{(z, x)}} \mathcal{R}(z, x, t) f(z, x, t),
\end{aligned}
$$

with $f \in \mathcal{C}_{\text {skew }}^{c}(\mathcal{R})$.

4.2. Symmetric case. We turn to the symmetric case. The symmetric exterior operator is the operator $d_{\text {sym }}^{1}: \mathcal{C}_{\text {sym }}^{c}(\mathcal{E}) \longrightarrow \mathcal{C}_{\text {sym }}^{c}(\mathcal{R})$, given by

$$
\forall f \in \mathcal{C}_{\text {sym }}^{c}(\mathcal{E}), \quad d_{\text {sym }}^{1}(f)(x, y, z)=f(x, y)+f(y, z)+f(z, x)
$$

The symmetric co-exterior derivative operator is the formal adjoint of $d_{\text {sym }}^{1}$, i.e. it is the operator $\delta_{\text {sym }}^{1}: \mathcal{C}_{\text {sym }}^{1}(\mathcal{R}) \longrightarrow \mathcal{C}_{\text {sym }}^{c}(\mathcal{E})$, given by

$$
\forall f \in \mathcal{C}_{\mathrm{sym}}^{1}(\mathcal{R}), \delta_{\mathrm{sym}}^{1}(f)(x, y):=\frac{1}{\mathcal{E}(x, y)} \sum_{z \in \mathcal{F}_{(x, y)}} \mathcal{R}(x, y, z) f(x, y, z) .
$$


Indeed, for $f \in \mathcal{C}_{\text {sym }}^{c}(\mathcal{E})$ and $g \in \mathcal{C}_{\text {sym }}^{c}(\mathcal{R})$ we get:

$$
\begin{aligned}
\left\langle d_{\mathrm{sym}}^{1} f, g\right\rangle & =\frac{1}{2} \sum_{(x, y, z) \in \mathcal{F}} \mathcal{R}(x, y, z) d_{\mathrm{sym}}^{1} f(x, y, z) \overline{g(x, y, z)} \\
& =\frac{1}{6} \sum_{x \sim y} \sum_{z \in \mathcal{F}_{(x, y)}} \mathcal{R}(x, y, z)(f(x, y)+f(y, z)+f(z, x) \overline{g(x, y, z)} \\
& =\frac{1}{2} \sum_{x \sim y} \sum_{z \in \mathcal{F}_{(x, y)}} \mathcal{R}(x, y, z) f(x, y) \overline{g(x, y, z)} \\
& =\frac{1}{2} \sum_{x \sim y} \mathcal{E}(x, y) f(x, y) \overline{\left(\frac{1}{\mathcal{E}(x, y)} \sum_{z \in \mathcal{F}_{(x, y)}} \mathcal{R}(x, y, z) g(x, y, z)\right)} \\
& =\left\langle f, \delta_{\mathrm{sym}}^{1} g\right\rangle .
\end{aligned}
$$

The operators $d_{\mathrm{sym}}^{1}$ and $\delta_{\mathrm{sym}}^{1}$ are closable. Indeed, since $\delta_{\mathrm{sym}}^{1}: \ell_{\mathrm{sym}}^{2}(\mathcal{R}) \longrightarrow$ $\ell_{\mathrm{sym}}^{2}(\mathcal{E})\left(\operatorname{resp} . d_{\mathrm{sym}}^{1}: \ell_{\mathrm{sym}}^{2}(\mathcal{E}) \longrightarrow \ell_{\mathrm{sym}}^{2}(\mathcal{R})\right)$ is with dense domain then $\delta_{\text {sym }}^{1}($ resp. $\left.d_{\text {sym }}^{1}\right)$ is closable. We denote their closure by the same symbol. The symmetric discrete Laplacian operator acting on 2 -forms is the operator $\mathcal{L}_{2, \text { sym }}=d_{\text {sym }}^{1} \delta_{\text {sym }}^{1}$, given by the same expression of $\mathcal{L}_{2, \text { skew }}$.

4.3. Relationship between $\mathcal{L}_{2 \text {,skew }}$ and $\mathcal{L}_{2 \text {,sym }}$. The two operators $\mathcal{L}_{2 \text {,skew }}$ and $\mathcal{L}_{2 \text {,sym }}$ have the same expression. However, they do not act on the same spaces. Namely, when $\mathcal{T}$ is tri-partite, we shall prove that the two operators are unitarily equivalent.

Definition 4.1. A tri-partite graph is a graph whose vertices can be partitioned into 3 disjoint sets so that there are no two vertices within the same set are adjacent. A tri-partite triangulation is a triangulation $\mathcal{T}=(\mathcal{G}, \mathcal{F})$ such that $\mathcal{G}$ is tri-partite.

Theorem 4.2. Let $\mathcal{T}=(\mathcal{V}, m, \mathcal{E}, \mathcal{R})$ be a tri-partite weighted triangulation. Then, $\mathcal{L}_{2, \text { skew }}$ and $\mathcal{L}_{2, \mathrm{sym}}$ are unitarily equivalent.

Proof. We consider the tri-partite decomposition $\left\{\mathcal{V}_{1}, \mathcal{V}_{2}, \mathcal{V}_{3}\right\}$. Set

$$
\circlearrowleft \mathcal{V}_{1} \times \mathcal{V}_{2} \times \mathcal{V}_{3}=\left\{\circlearrowleft(x, y, z):(x, y, z) \in \mathcal{V}_{1} \times \mathcal{V}_{2} \times \mathcal{V}_{3}\right\}
$$

Let $\mathcal{U}: \ell_{\text {skew }}^{2}(\mathcal{R}) \longrightarrow \ell_{\text {sym }}^{2}(\mathcal{R})$ be the unitary map given by

$$
\mathcal{U}(f)(x, y, z)=S(x, y, z) f(x, y, z),
$$

where

$$
S(x, y, z):=\left\{\begin{aligned}
1, & \text { if }(x, y, z) \in \circlearrowleft \mathcal{V}_{1} \times \mathcal{V}_{2} \times \mathcal{V}_{3}, \\
-1, & \text { if }(x, y, z) \in \circlearrowleft \mathcal{V}_{3} \times \mathcal{V}_{2} \times \mathcal{V}_{1}
\end{aligned}\right.
$$


Let $\mathcal{W}$ be the following mapping from $\ell_{\text {sym }}^{2}(\mathcal{R})$ into $\ell_{\text {skew }}^{2}(\mathcal{R}): \mathcal{W}(f)(x, y, z)=$ $S(x, y, z) f(x, y, z)$. Then

$$
\langle\mathcal{U} f, g\rangle=\langle f, \mathcal{W} g\rangle, \mathcal{U}(\mathcal{W}(g))=g \text { and } \mathcal{W}(\mathcal{U}(f))=f
$$

for all $f \in \ell_{\text {skew }}^{2}(\mathcal{F})$ and $g \in \ell_{\text {sym }}^{2}(\mathcal{F})$. So we have

$$
\mathcal{W}(f)=\mathcal{U}^{-1}(f)=\mathcal{U}^{*}(f)
$$

for all $f \in \ell_{\text {sym }}^{2}(\mathcal{R})$. Therefore,

$$
\mathcal{U} \mathcal{L}_{2, \text { skew }} \mathcal{U}^{-1}(f)(x, y, z)=\mathcal{L}_{2, \text { sym }}(f)(x, y, z)
$$

for all $f \in \mathcal{C}_{\text {sym }}^{c}(\mathcal{R})$.

\section{A Nelson CRiterium}

For the general theory of unbounded Hermitian operators and their extensions, we refer the reader to $[25,20,27]$. Let $\mathcal{L}_{\text {skew }}$ be the following mapping from $\mathcal{C}_{\text {skew }}(\mathcal{R})$ into itself:

$$
\begin{aligned}
\mathcal{L}_{\text {skew }}(f)(x, y, z) & =\frac{1}{\mathcal{E}(x, y)} \sum_{t \in \mathcal{F}_{(x, y)}} \mathcal{R}(x, y, t) f(x, y, t) \\
& +\frac{1}{\mathcal{E}(y, z)} \sum_{t \in \mathcal{F}_{(y, z)}} \mathcal{R}(y, z, t) f(y, z, t) \\
& +\frac{1}{\mathcal{E}(z, x)} \sum_{t \in \mathcal{F}_{(z, x)}} \mathcal{R}(z, x, t) f(z, x, t) .
\end{aligned}
$$

Let $\mathcal{L}_{2, \text { max skew }}$ be the restrictions of $\mathcal{L}_{\text {skew }}$ to

$$
\mathcal{D}\left(\mathcal{L}_{2, \text { max }, \text { skew }}\right):=\left\{f \in \ell_{\text {skew }}^{2}(\mathcal{R}) \text { such that } \mathcal{L}_{\text {skew }} f \in \ell_{\text {skew }}^{2}(\mathcal{R})\right\} .
$$

Lemma 5.1. $\mathcal{L}_{2, \text { skew }}^{*}=\mathcal{L}_{2, \text { max }, \text { skew }}$.

Proof. Let $f \in \mathcal{C}_{\text {skew }}^{c}(\mathcal{R})$ and let $g \in \mathcal{C}_{\text {skew }}(\mathcal{R})$. Let $\mathcal{F}_{00}$ the support of $f$ and set

$$
\mathcal{F}_{0}=\left\{(x, y, z) \in \mathcal{F}: \quad \exists u \in \mathcal{V},\{(x, y, u), \quad(y, z, u), \quad(z, x, u)\} \cap \mathcal{F}_{00} \neq \emptyset\right\}
$$


which is a finite set. Then, $\operatorname{supp}\left(\mathcal{L}_{2, \text { skew }}\right) \subset \mathcal{F}_{0}$ and the following relation holds:

$$
\begin{aligned}
& \frac{1}{2} \sum_{(x, y, z) \in \mathcal{F}_{0}} \mathcal{R}(x, y, z) \mathcal{L}_{2, \text { skew }}(f)(x, y, z) \overline{g(x, y, z)}= \\
& \frac{1}{2} \sum_{(x, y, z) \in \mathcal{F}_{0}} \mathcal{R}(x, y, z)\left(\frac{1}{\mathcal{E}(x, y)} \sum_{t \in \mathcal{F}_{(x, y)}} \mathcal{R}(x, y, t) f(x, y, t)\right. \\
& +\frac{1}{\mathcal{E}(y, z)} \sum_{t \in \mathcal{F}_{(y, z)}} \mathcal{R}(y, z, t) f(y, z, t) \\
& \left.+\frac{1}{\mathcal{E}(z, x)} \sum_{t \in \mathcal{F}_{(z, x)}} \mathcal{R}(z, x, t) f(z, x, t)\right) \overline{g(x, y, z)} \\
& =\frac{1}{2} \sum_{(x, y, z) \in \mathcal{F}_{00}} \mathcal{R}(x, y, z) f(x, y, z) \overline{\left(\frac{1}{\mathcal{E}(x, y)} \sum_{u \in \mathcal{V}} \mathcal{R}(x, y, u) g(x, y, u)\right.} \\
& +\overline{\frac{1}{\mathcal{E}(y, z)} \sum_{u \in \mathcal{V}} \mathcal{R}(y, z, u) g(y, z, u)} \\
& \left.+\overline{\frac{1}{\mathcal{E}(z, x)} \sum_{u \in \mathcal{V}} \mathcal{R}(z, x, u) g(z, x, u)}\right) \\
& =\frac{1}{2} \sum_{x, y, z \in \mathcal{V}} \mathcal{R}(x, y, z) f(x, y, z) \overline{\mathcal{L}_{2} g(x, y, z)} .
\end{aligned}
$$

Let $g \in \mathcal{D}\left(\mathcal{L}_{2, \text { max ,skew }}\right)$. It follows from (1) that

$$
\left\langle\mathcal{L}_{2, \text { skew }} f, g\right\rangle=\left\langle f, \mathcal{L}_{2, \text { max }, \text { skew }} g\right\rangle
$$

for all $f \in \mathcal{C}_{\text {skew }}^{c}(\mathcal{R})$, which implies that $g \in \mathcal{D}\left(\mathcal{L}_{2, \text { skew }}^{*}\right)$. Now let $g \in$ $\mathcal{D}\left(\mathcal{L}_{2, \text { skew }}^{*}\right)$. Let $(x, y, z) \in \mathcal{F}$ and let

$$
f=\frac{1}{\mathcal{R}(x, y, z)}\left(\mathbf{1}_{\circlearrowleft(x, y, z)}-\mathbf{1}_{\circlearrowleft(z, y, x)}\right) .
$$

Then, $f \in \mathcal{C}_{\text {skew }}^{c}(\mathcal{R})$ and we obtain from $(1)$ :

$$
\begin{aligned}
\overline{\left(\mathcal{L}_{2, \text { skew }}^{*} g\right)(x, y, z)} & =\left\langle f, \mathcal{L}_{2, \text { skew }}^{*} g\right\rangle \\
& =\left\langle\mathcal{L}_{2, \text { skew }} f, g\right\rangle \\
& =\frac{1}{2} \sum_{(u, v, w) \in \mathcal{F}} \mathcal{R}(u, v, w) \mathcal{L}_{2, \text { skew }} f(u, v, w) \overline{g(u, v, w)} \\
& =\frac{1}{2} \sum_{(u, v, w) \in \mathcal{F}} \mathcal{R}(u, v, w) f(u, v, w) \overline{\mathcal{L}_{2, \text { max }, \text { skew }} g(u, v, w)} \\
& =\frac{\left(\mathcal{L}_{2, \text { max }, \text { skew }} g\right)(x, y, z)}{}
\end{aligned}
$$


which implies that $\mathcal{L}_{2 \text {,skew }} g=\mathcal{L}_{2 \text {,max,skew }} g \in \ell_{\text {skew }}^{2}(\mathcal{R})$ by the definition of the adjoint, it follows that $g \in \mathcal{D}\left(\mathcal{L}_{2 \text {,max,skew }}\right)$. Hence $\mathcal{L}_{2 \text {,skew }}^{*}=\mathcal{L}_{2 \text {,max,skew }}$.

Remark 5.2. Let $\mathcal{L}_{\text {sym }}$ be the mapping from $\mathcal{C}_{\text {sym }}(\mathcal{R})$ into itself given by the same expression of $\mathcal{L}_{2, \mathrm{sym}}$. Then, $\mathcal{L}_{2, \mathrm{sym}}^{*}=\mathcal{L}_{2 \text {,max,sym }}$ where $\mathcal{L}_{2 \text {,max,sym }}$ is the restrictions of $\mathcal{L}_{\mathrm{sym}}$ to

$$
\mathcal{D}\left(\mathcal{L}_{2, \text { max }, \text { sym }}\right):=\left\{f \in \ell_{\text {sym }}^{2}(\mathcal{R}) \text { such that } \mathcal{L}_{\text {sym }} f \in \ell_{\text {sym }}^{2}(\mathcal{R})\right\} .
$$

Using the Nelson commutator theorem, we prove the criterium of essential self-adjointness for $\mathcal{L}_{2 \text {,skew }}$ and $\mathcal{L}_{2 \text {,sym }}$.

Theorem 5.3. Let $\mathcal{T}=(\mathcal{V}, m, \mathcal{E}, \mathcal{R})$ be a weighted triangulation. Set

$$
\mathcal{N}(x, y, z)=1+d_{\mathcal{E}}(x, y)+d_{\mathcal{E}}(y, z)+d_{\mathcal{E}}(z, x) .
$$

Suppose that

$$
\sup _{x \sim y, z \in \mathcal{F}_{(x, y)}} \sum_{r \in \mathcal{F}_{(x, y)}} \frac{1}{\mathcal{E}(x, y)} \mathcal{R}(x, y, r)|\mathcal{N}(x, y, r)-\mathcal{N}(x, y, z)|^{2}<\infty .
$$

Then $\mathcal{L}_{2, \text { skew }}$ is essentially self-adjoint on $\mathcal{C}_{\text {skew }}^{c}(\mathcal{R})$ and $\mathcal{L}_{2 \text {,sym }}$ is essentially self-adjoint on $\mathcal{C}_{\text {sym }}^{c}(\mathcal{R})$.

Proof. Let $\mathcal{N}$ be the operator of multiplication by $\mathcal{N}(., .,$.$) ant take f \in$ $\mathcal{C}_{\text {skew }}^{c}(\mathcal{F})$. Going over the same techniques of the proof of [3, Theorem 5.13], we obtain:

$$
\begin{aligned}
\left\|\mathcal{L}_{2, \text { skew }} f\right\|^{2} & \leq \frac{2}{3} \sum_{x \sim y, z \in \mathcal{F}_{(x, y)}} \mathcal{R}(x, y, z)\left(\frac{1}{\mathcal{E}^{2}(x, y)}\left|\sum_{t \in \mathcal{F}_{(x, y)}} \mathcal{R}(x, y, t) f(x, y, t)\right|^{2}\right. \\
& +\frac{1}{\mathcal{E}^{2}(y, z)}\left|\sum_{t \in \mathcal{F}_{(y, z)}} \mathcal{R}(y, z, t) f(y, z, t)\right|^{2} \\
& \left.+\frac{1}{\mathcal{E}^{2}(z, x)}\left|\sum_{t \in \mathcal{F}_{(z, x)}} \mathcal{R}(z, x, t) f(z, x, t)\right|^{2}\right) \\
& \leq 2 \sum_{x \sim y, z \in \mathcal{F}_{(x, y)}} \mathcal{R}(x, y, z) \frac{1}{\mathcal{E}^{2}(x, y)}\left|\sum_{t \in \mathcal{F}_{(x, y)}} \mathcal{R}(x, y, t) f(x, y, t)\right|^{2} \\
& \leq 2 \sum_{x \sim y, z \in \mathcal{F}_{(x, y)}} \mathcal{R}(x, y, z) \frac{1}{\mathcal{E}^{2}(x, y)}\left(\sum_{r \in \mathcal{F}_{(x, y)}} \mathcal{R}(x, y, r)\right) \times \\
& =2 \sum_{x \sim y, z \in \mathcal{F}_{(x, y)}} \mathcal{R}(x, y, z)\left(\frac{1}{\mathcal{R}(x, y)} \sum_{t \in \mathcal{F}_{(x, y)}} \mathcal{R}(x, y, t)|f(x, y, t)|^{2}\right) \\
& \leq 12\|\mathcal{N}(f)\|^{2} .
\end{aligned}
$$


Moreover, we notice that $\mathcal{N}(., .,$.$) is symmetric and f(x, y, z)=-f(z, y, x)$ and let $J=\left|\left\langle f,\left[\mathcal{L}_{2, \text { skew }}, \mathcal{N}\right] f\right\rangle\right|$. We get:

$$
\begin{aligned}
& J \leq \frac{1}{12} \sum_{x \sim y, z \in \mathcal{F}_{(x, y)}} \mathcal{R}(x, y, z)\left(|f(x, y, z)|^{2}\right. \\
& \left.+\left|\left[\mathcal{L}_{2, \text { skew }}, \mathcal{N}\right](f)(x, y, z)\right|^{2}\right) \\
& \leq \frac{1}{2}\left\|\mathcal{N}^{\frac{1}{2}}(f)\right\|^{2}+\sum_{x \sim y, z \in \mathcal{F}_{(x, y)}} \mathcal{R}(x, y, z) \mid \sum_{t \in \mathcal{F}_{(x, y)}} \frac{1}{\mathcal{E}(x, y)} \mathcal{R}(x, y, t) \times \\
& \left.(\mathcal{N}(x, y, t)-\mathcal{N}(x, y, z)) f(x, y, t)\right|^{2} \\
& \leq \frac{1}{2}\left\|\mathcal{N}^{\frac{1}{2}}(f)\right\|^{2}+\sum_{x \sim y, z \in \mathcal{F}_{(x, y)}} \mathcal{R}(x, y, z)\left(\sum_{t \in \mathcal{F}_{(x, y)}} \frac{1}{\mathcal{E}(x, y)} \mathcal{R}(x, y, t)\right) \times \\
& \left(\sum_{r \in \mathcal{F}_{(x, y)}} \frac{1}{\mathcal{E}(x, y)} \mathcal{R}(x, y, r)|\mathcal{N}(x, y, r)-\mathcal{N}(x, y, z)|^{2}|f(x, y, r)|^{2}\right) \\
& =\frac{1}{2}\left\|\mathcal{N}^{\frac{1}{2}}(f)\right\|^{2}+\sum_{x \sim y, z \in \mathcal{F}_{(x, y)}} \mathcal{R}(x, y, z)\left(\sum_{t \in \mathcal{F}_{(x, y)}} \frac{1}{\mathcal{E}(x, y)} \mathcal{R}(x, y, t)\right) \times \\
& \underbrace{\sum_{r \in \mathcal{V}} \frac{1}{\mathcal{E}(x, y)} \mathcal{F}(x, y, r)|\mathcal{N}(x, y, r)-\mathcal{N}(x, y, z)|^{2}}_{\leq C}|f(x, y, z)|^{2} \\
& \leq\left(\frac{1+12 C}{2}\right)\left\|\mathcal{N}^{\frac{1}{2}}(f)\right\| .
\end{aligned}
$$

Applying [26, Theorem X.37], the result follows. The proof of $\mathcal{L}_{2, \text { sym }}$ may be checked in the same way as the proof of $\mathcal{L}_{2 \text {,skew }}$.

Corollary 5.4. Let $\mathcal{T}=(\mathcal{V}, m, \mathcal{E}, \mathcal{R})$ be a simple triangulation. Assume that

$$
\sup _{x \sim y, z \in \mathcal{F}_{(x, y)}} \sum_{r \in \mathcal{F}_{(x, y)}}\left|\sharp \mathcal{F}_{(y, z)}+\sharp \mathcal{F}_{(z, x)}-\sharp \mathcal{F}_{(y, r)}-\sharp \mathcal{F}_{(r, x)}\right|^{2}<\infty .
$$

Then $\mathcal{L}_{2, \text { skew }}$ is essentially self-adjoint on $\mathcal{C}_{\text {skew }}^{c}(\mathcal{R})$ and $\mathcal{L}_{2 \text {,sym }}$ is essentially self-adjoint on $\mathcal{C}_{\text {sym }}^{c}(\mathcal{R})$.

\section{Essential SPECTRUm}

Let $A$ be a closed, densely defined linear operator on a Banach space $X$, and let $\sigma(A)$ denote the spectrum of $A$. We denote by $\mathcal{K}(X)$ the set of compact operators on $X$ to itself. We define the essential spectrum of the operator $A$ by

$$
\sigma_{\mathrm{ess}}(A)=\bigcap_{K \in \mathcal{K}(X)} \sigma(A+K) .
$$


It is well known that if $A$ is a self-adjoint operator on a Hilbert space, the essential spectrum of $A$ is the set of limit points of the spectrum of $A$, i.e., all points of the spectrum except isolated eigenvalues of finite multiplicity, see [30]. Let $\mathcal{T}=(\mathcal{V}, m, \mathcal{E}, \mathcal{R})$ be a weighted triangulation. Note that $\mathcal{L}_{2 \text {,skew }}$ is non-negative symmetric operator on $\mathcal{C}_{\text {skew }}^{c}(\mathcal{R})$. We consider the quadratic form

$$
q(f, g)=\left\langle f, \mathcal{L}_{2, \mathrm{skew}} g\right\rangle+\langle f, g\rangle
$$

on $\mathcal{C}_{\text {skew }}^{c}(\mathcal{R}) \times \mathcal{C}_{\text {skew }}^{c}(\mathcal{R})$. Let $\mathcal{H}_{1}$ be the completion of $\mathcal{C}_{\text {skew }}^{c}(\mathcal{R})$ under the norm

$$
\|f\|_{q}=\sqrt{\left\langle\mathcal{L}_{2, \mathrm{skew}} f, f\right\rangle+\|f\|^{2}} .
$$

We define the Friedrichs extension $\mathcal{L}_{2 \text {,skew }}^{\mathcal{F}}$ of $\mathcal{L}_{2 \text {,skew }}$ by:

i) A vector $f$ is in domain $\mathcal{D}\left(\mathcal{L}_{2 \text {,skew }}^{\mathcal{F}}\right)$ if and only if $f \in \mathcal{H}_{1}$ and $\mathcal{C}_{\text {skew }}^{c}(\mathcal{R}) \ni$ $g \longmapsto\left\langle f, \mathcal{L}_{2, \text { skew }} g\right\rangle+\langle f, g\rangle$ extends to a norm continuous function on $\ell_{\text {skew }}^{2}(\mathcal{R})$. (ii) For each $f \in \mathcal{D}\left(\mathcal{L}_{2, \text { skew }}^{\mathcal{F}}\right)$, there is a unique $u_{f}$ such that $\left\langle f, \mathcal{L}_{2, \text { skew }} g\right\rangle+$ $\langle f, g\rangle=\left\langle u_{f}, g\right\rangle$ by Riesz' Theorem. The Friedrichs extension of $\mathcal{L}_{2, \text { skew }}$, is given by $\mathcal{L}_{2 \text {,skew }}^{\mathcal{F}} f=u_{f}-f$. It is a self-adjoint extension of $\mathcal{L}_{2 \text {,skew }}$, e.g. see [26, Theorem X.23]. Note that $\mathcal{L}_{2 \text {,skew }}^{\mathcal{F}}$ is bounded if and only if $d_{\mathcal{E}}($.$) is$ bounded, e.g. see [8].

Theorem 6.1. Let $\mathcal{T}=(\mathcal{V}, m, \mathcal{E}, \mathcal{R})$ be a weighted triangulation and let $\mathcal{F}_{0}=\{\mathcal{K} \subset \mathcal{F}: \mathcal{K}$ finite $\}$. Then,

$$
\inf \sigma\left(\mathcal{L}_{2, \text { skew }}^{\mathcal{F}}\right) \leq \inf _{(x, y, z) \in \mathcal{F}} \mathcal{R}(x, y, z)\left(\frac{1}{\mathcal{E}(x, y)}+\frac{1}{\mathcal{E}(y, z)}+\frac{1}{\mathcal{E}(z, x)}\right) .
$$

and

$$
\inf \sigma_{\text {ess }}\left(\mathcal{L}_{2, \text { skew }}^{\mathcal{F}}\right) \leq \sup _{\mathcal{K} \subset \mathcal{F}_{0}} \inf _{(x, y, z) \in \mathcal{K}^{c}} \mathcal{R}(x, y, z)\left(\frac{1}{\mathcal{E}(x, y)}+\frac{1}{\mathcal{E}(y, z)}+\frac{1}{\mathcal{E}(z, x)}\right) .
$$

In particular, if $\mathcal{T}$ is a simple triangulation then $\mathcal{L}_{2, \text { skew }}^{\mathcal{F}}$ is not with compact resolvent.

Proof. Let $\left(x_{0}, y_{0}, z_{0}\right) \in \mathcal{F}$ and let

$$
f=\frac{\mathbf{1}_{\circlearrowleft\left(x_{0}, y_{0}, z_{0}\right)}-\mathbf{1}_{\circlearrowleft\left(z_{0}, y_{0}, x_{0}\right)}}{\sqrt{\mathcal{R}\left(x_{0}, y_{0}, z_{0}\right)}}
$$

where $\mathbf{1}_{\circlearrowleft\left(x_{0}, y_{0}, z_{0}\right)}$ denotes the indicator function of $\circlearrowleft\left(x_{0}, y_{0}, z_{0}\right)$. Then $\|f\|=1$ and

$$
\left\langle f, \mathcal{L}_{2, \text { skew }} f\right\rangle=\mathcal{R}\left(x_{0}, y_{0}, z_{0}\right)\left(\frac{1}{\mathcal{E}\left(x_{0}, y_{0}\right)}+\frac{1}{\mathcal{E}\left(y_{0}, z_{0}\right)}+\frac{1}{\mathcal{E}\left(z_{0}, x_{0}\right)}\right)
$$

Applying [21, Proposition 3], the result follows. 


\section{Applichtion to The STUdy of the ADJACENCY MATRIX}

7.1. Adjacency matrix. The adjacency matrix has important implications. For example, it uses the semi-boundedness in order to give meaning to the heat equation, see [5]. Let $\mathcal{G}=(\mathcal{V}, m, \mathcal{E})$ be a weighted graph. We define the set of 0 -cochains on $\mathcal{V}$ by

$$
\mathcal{C}(\mathcal{V})=\{f: \mathcal{V} \longrightarrow \mathbb{C}\}
$$

We denote by $\mathcal{C}^{c}(\mathcal{V})$ the 0 -cochains with finite support in $\mathcal{V}$. We associate a Hilbert space to $\mathcal{V}$ :

$$
\ell^{2}(\mathcal{V})=\left\{f \in \mathcal{C}(\mathcal{V}) \text { such that }\|f\|^{2}=\sum_{x \in \mathcal{V}} m(x)|f(x)|^{2}<\infty\right\} .
$$

The associated scalar product is given by

$$
\langle f, g\rangle=\sum_{x \in \mathcal{V}} m(x) \overline{f(x)} g(x), \text { for } f, g \in \ell^{2}(\mathcal{V}) .
$$

We define the adjacency matrix:

$$
\mathcal{A}_{\mathcal{G}}(f)(x)=\frac{1}{m(x)} \sum_{y \in \mathcal{V}} \mathcal{E}(x, y) f(y), f \in \mathcal{C}^{c}(\mathcal{V}) .
$$

It is symmetric and thus closable. We denote its closure by the same symbol. When $\mathcal{G}$ is simple, we have that $\mathcal{A}_{\mathcal{G}}$ is unbounded if and only if it is unbounded from above and if and only if the degree is unbounded, see [15].

7.2. Triangular graph. Let $\mathcal{T}=(\mathcal{V}, m, \mathcal{E}, \mathcal{R})$ be a weighted triangulation. Set $\widehat{\mathcal{V}}=\mathcal{F} / \sim$, where $\varpi \sim-\varpi$.

Definition 7.1. Let $\mathcal{T}=(\mathcal{V}, m, \mathcal{E}, \mathcal{R})$ be a weighted triangulation. Set $\widehat{\mathcal{V}}=$ $\mathcal{F} / \sim$, where $\varpi \sim-\varpi$. The triangular graph of $\mathcal{T}$ is the graph $\widehat{\mathcal{G}}=(\widehat{\mathcal{V}}, \widehat{m}, \widehat{\mathcal{E}})$ where $\widehat{m}=1$ and

$$
\begin{aligned}
\widehat{\mathcal{E}}\left(\left(x_{0}, y_{0}, z_{0}\right),(x, y, z)\right) & =\sqrt{\mathcal{R}\left(x_{0}, y_{0}, z_{0}\right)}\left(\frac{\sqrt{\mathcal{R}(x, y, z)}}{\mathcal{E}(x, y)} 1_{x=x_{0}, y=y_{0}}\right. \\
& \left.+\frac{\sqrt{\mathcal{R}(x, y, z)}}{\mathcal{E}(y, z)} 1_{y=y_{0}, z=z_{0}}+\frac{\sqrt{\mathcal{R}(x, y, z)}}{\mathcal{E}(z, x)} 1_{z=z_{0}, x=x_{0}}\right)
\end{aligned}
$$

if $\left(x_{0}, y_{0}, z_{0}\right) \neq(x, y, z)$ and 0 otherwise.

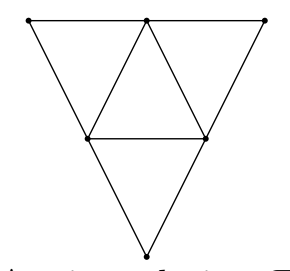

A triangulation $\mathcal{T}$

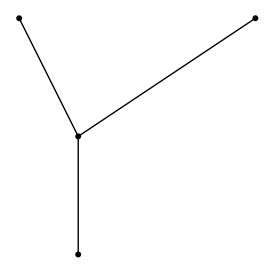

Triangular graph $\widehat{\mathcal{G}}$ 
Remark 7.2. Let $\mathcal{T}=(\mathcal{V}, m, \mathcal{E}, \mathcal{R})$ be a triangulation. The adjacency matrix on $\widehat{\mathcal{G}}$ is given by

$$
\begin{aligned}
\mathcal{A}_{\widehat{\mathcal{G}}}(f)\left(x_{0}, y_{0}, z_{0}\right):= & \sum_{z \in \mathcal{V}, z \neq z_{0}} \frac{\sqrt{\mathcal{R}\left(x_{0}, y_{0}, z_{0}\right) \mathcal{R}\left(x_{0}, y_{0}, z\right)}}{\mathcal{E}\left(x_{0}, y_{0}\right)} f\left(x_{0}, y_{0}, z\right) \\
& +\sum_{x \in \mathcal{V}, x \neq x_{0}} \frac{\sqrt{\mathcal{R}\left(x_{0}, y_{0}, z_{0}\right) \mathcal{R}\left(x, y_{0}, z_{0}\right)}}{\mathcal{E}\left(y_{0}, z_{0}\right)} f\left(x, y_{0}, z_{0}\right) \\
& +\sum_{y \in \mathcal{V}, y \neq y_{0}} \frac{\sqrt{\mathcal{R}\left(x_{0}, y_{0}, z_{0}\right) \mathcal{R}\left(x_{0}, y, z_{0}\right)}}{\mathcal{E}\left(z_{0}, x_{0}\right)} f\left(x_{0}, y, z_{0}\right)
\end{aligned}
$$

for all $f \in \mathcal{C}^{c}(\widehat{\mathcal{V}})$.

Proposition 7.3. Let $\mathcal{T}=(\mathcal{V}, m, \mathcal{E}, \mathcal{R})$ be a weighted triangulation. Then $\mathcal{L}_{2, \text { sym }}$ is unitarily equivalent to

$$
\mathcal{A}_{\widehat{\mathcal{G}}}+\mathcal{Q}(V)
$$

where

$$
V\left(x_{0}, y_{0}, z_{0}\right)=\frac{\mathcal{R}\left(x_{0}, y_{0}, z_{0}\right)}{\mathcal{R}\left(x_{0}, y_{0}\right)}+\frac{\mathcal{R}\left(x_{0}, y_{0}, z_{0}\right)}{\mathcal{E}\left(y_{0}, z_{0}\right)}+\frac{\mathcal{R}\left(x_{0}, y_{0}, z_{0}\right)}{\mathcal{E}\left(z_{0}, x_{0}\right)}
$$

and $\mathcal{Q}(V)$ be the operator of multiplication by $V$.

Proof. Set $U: \ell_{\text {sym }}^{2}(\mathcal{R}) \longrightarrow \ell^{2}(\widehat{\mathcal{V}})$ as the operator given by

$$
U(f)(x, y, z)=\sqrt{\mathcal{R}(x, y, z)} f(x, y, z) .
$$

Notice that

$$
U^{-1}(f)(x, y, z)=U^{*}(f)(x, y, z)=\frac{1}{\sqrt{\mathcal{R}(x, y, z)}} f(x, y, z)
$$

for all $f \in \ell^{2}(\widehat{\mathcal{V}})$. Notice now that on $\mathcal{C}_{c}(\widehat{\mathcal{V}})$

$$
\begin{aligned}
U \mathcal{L}_{2, \mathrm{sym}} U^{-1}(f)\left(x_{0}, y_{0}, z_{0}\right) & =\sum_{t \in \mathcal{V}} \frac{\sqrt{\mathcal{R}\left(x_{0}, y_{0}, z_{0}\right) \mathcal{R}\left(x_{0}, y_{0}, t\right)}}{\mathcal{E}\left(x_{0}, y_{0}\right)} f\left(x_{0}, y_{0}, t\right) \\
& +\sum_{t \in \mathcal{V}} \frac{\sqrt{\mathcal{R}\left(x_{0}, y_{0}, z_{0}\right) \mathcal{R}\left(y_{0}, z_{0}, t\right)}}{\mathcal{E}\left(y_{0}, z_{0}\right)} f\left(y_{0}, z_{0}, t\right) \\
& +\sum_{t \in \mathcal{V}} \frac{\sqrt{\mathcal{R}\left(x_{0}, y_{0}, z_{0}\right) \mathcal{R}\left(z_{0}, x_{0}, t\right)}}{\mathcal{E}\left(z_{0}, x_{0}\right)} f\left(z_{0}, x_{0}, t\right) .
\end{aligned}
$$

Using Remark 7.2, we obtain the result. 
Corollary 7.4. Let $\mathcal{T}=(\mathcal{V}, m, \mathcal{E}, \mathcal{R})$ be a weighted 3 -partite triangulation. If $V$ is bounded on $\mathcal{F}$, then

$$
\mathcal{A}_{\widehat{\mathcal{G}}} \geq \sup _{(x, y, z) \in \mathcal{F}} V(x, y, z) .
$$

In particular, $\mathcal{A}_{\widehat{\mathcal{G}}} \geq-3$ when $\mathcal{T}$ is simple.

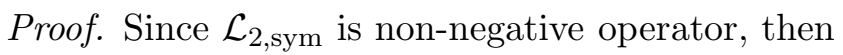

$$
\left\langle\mathcal{A}_{\widehat{\mathcal{G}}} f, f\right\rangle \geq-3\|f\|^{2}
$$

for all $f \in \mathcal{C}^{c}(\widehat{\mathcal{V}})$

Corollary 7.5. Let $\mathcal{T}=(\mathcal{V}, m, \mathcal{E}, \mathcal{R})$ be a 3 -partite weighted triangulation. Set

$$
\mathcal{N}(x, y, z)=1+\sum_{r \in \mathcal{V}}\left(d_{\mathcal{E}}(x, y)+d_{\mathcal{E}}(y, z)+d_{\mathcal{E}}(z, x)\right)
$$

Suppose that

$$
\sup _{(x, y, z) \in \mathcal{F}} \sum_{r \in \mathcal{F}_{(x, y)}} \frac{1}{\mathcal{E}(x, y)} \mathcal{R}(x, y, r)|\mathcal{N}(x, y, r)-\mathcal{N}(x, y, z)|^{2}<\infty .
$$

and $V$ is bounded. Then, $\mathcal{A}_{\widehat{\mathcal{G}}}$ is essentially self-adjoint on $\mathcal{C}^{c}(\widehat{\mathcal{V}})$.

Proof. Combine Proposition 7.3, Theorem 5.3 and Theorem 4.2.

7.3. Geometric Hypothesis. We recall the two following definitions:

Definition 7.6. $[1$, Definition 8] The graph $\mathcal{G}:=(\mathcal{V}, m, \mathcal{E})$ is $\chi$-complete if there exists an increasing sequence of finite set $\left(\mathcal{V}_{n}\right)_{n}$ such that $\mathcal{V}=\cup_{n} \mathcal{V}_{n}$ and there exist related functions $\chi_{n}$ satisfying the following three conditions:

1) $\chi_{n} \in \mathcal{C}^{c}(\mathcal{V}), 0 \leq \chi_{n} \leq 1$,

2) $\chi_{n}(x)=1$ if $x \in \mathcal{V}_{n}$,

3) $\exists C>0, \forall n \in \mathbb{N}, x \in \mathcal{V}$,

$$
\frac{1}{m(x)} \sum_{y \in \mathcal{V}} \mathcal{E}(x, y)\left|\chi_{n}(x)-\chi_{n}(y)\right|^{2} \leq C .
$$

Definition 7.7. [7, Definition 4.2] A weighted triangulation $\mathcal{T}=(\mathcal{V}, m, \mathcal{E}, \mathcal{R})$ is $\chi$-complete, if

1) $\mathcal{G}=(\mathcal{V}, m, \mathcal{E})$ is $\chi$-complete.

2) $\exists M>0, \forall n \in \mathbb{N},(x, y) \in \mathcal{E}$

$$
\frac{1}{\mathcal{E}(x, y)} \sum_{t \in \mathcal{F}_{(x, y)}} \mathcal{R}(x, y, t)\left|2 \chi_{n}(t)-\chi_{n}(x)-\chi_{n}(y)\right|^{2} \leq M .
$$

We recall the criterion obtained in [7].

Theorem 7.8. Let $\mathcal{T}=(\mathcal{V}, m, \mathcal{E}, \mathcal{R})$ be a $\chi$-complete weighted triangulation then $\mathcal{L}_{2 \text {,skew }}$ is essentially self-adjoint on $\mathcal{C}_{\text {skew }}^{c}(\mathcal{R})$. 
Corollary 7.9. Let $\mathcal{T}=(\mathcal{V}, m, \mathcal{E}, \mathcal{R})$ be a $\chi$-complete weighted triangulation. If $\mathcal{T}$ is tri-partite then $\mathcal{A}_{\widehat{\mathcal{G}}}$ is essentially self-adjoint on $\mathcal{C}^{c}(\widehat{\mathcal{V}})$.

Proof. Combine Proposition 7.3, Theorem 4.2 and Theorem 7.8.

7.4. Book-like triangulation. We recall the definition of 1 -dimensional decomposition given in [6] for the case of graphs.

Definition 7.10. [6] A 1-dimensional decomposition of the graph $\mathcal{G}=$ $(\mathcal{V}, m, \mathcal{E})$ is a family of finite sets $\left(S_{n}\right)_{n \in \mathbb{N}}$ which forms a partitions of $\mathcal{V}$, that is $\mathcal{V}=\cup_{n \in \mathbb{N}} S_{n}$, and such that for all $x \in S_{n}, y \in S_{m}$,

$$
\mathcal{E}(x, y)>0 \Longrightarrow|n-m| \leq 1
$$

The following definition is introduced in [7].

Definition 7.11. Let $\mathcal{T}:=(\mathcal{V}, m, \mathcal{E}, \mathcal{R})$ be a weighted triangulation and $\left(S_{n}\right)_{n \in \mathbb{N}}$ a 1-dimensional decomposition of the graph $\mathcal{G}=(\mathcal{V}, m, \mathcal{E}) . W e$ say that $\mathcal{T}$ is a book-like triangulation if

1) $\sharp S_{0}=1, \sharp S_{2 n+1}=2$ and $\sharp\left(S_{2 n+1}^{2} \cap \mathcal{E}\right)=1$, for all $n \in \mathbb{N}$.

2) $x, y \in S_{2 n+2} \Longrightarrow \mathcal{E}(x, y)=0$,

3) $\forall x \in S_{2 n+1}, \mathcal{N}_{\mathcal{G}}(x)=S_{2 n} \cup S_{2 n+2}$.

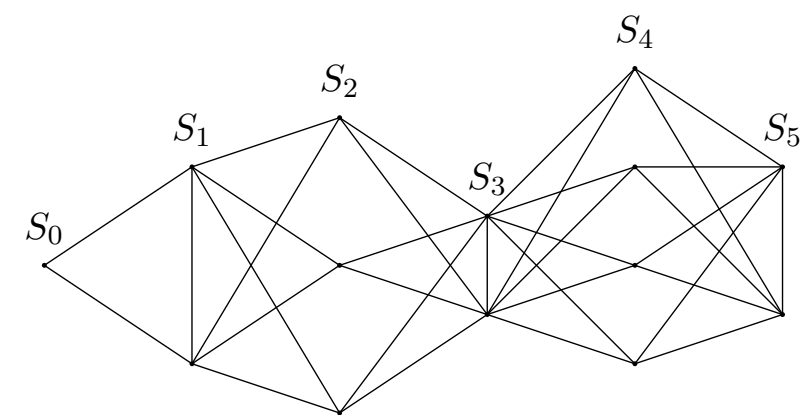

A book-like triangulation

We recall [7, Proposition 6.5]:

Proposition 7.12. Let $\mathcal{T}$ be a simple book-like triangulation. Assume that

$$
n \longmapsto \frac{\sharp S_{2 n}}{\sharp S_{2(n+1)}} \in \ell^{1}(\mathbb{N}) .
$$

Then, $\mathcal{L}_{2, \text { skew }}$ is not essentially self-adjoint on $\mathcal{C}_{\text {skew }}^{c}(\mathcal{R})$.

Corollary 7.13. Let $\mathcal{T}=(\mathcal{V}, m, \mathcal{E}, \mathcal{R})$ be a simple book-like triangulation satisfying (2). Then, $\mathcal{A}_{\widehat{\mathcal{G}}}$ is not essentially self-adjoint on $\mathcal{C}^{c}(\widehat{\mathcal{V}})$.

Proof. Set $S_{0}=\left\{x_{0}^{0}\right\}, S_{2 n+1}=\left\{x_{n}^{0}, x_{n}^{1}\right\}, \mathcal{V}_{0}=\cup_{n} S_{2 n}, \mathcal{V}_{1}=\cup_{n}\left\{x_{n}^{0}: n \in \mathbb{N}\right\}$ and $\mathcal{V}_{2}=\cup_{n}\left\{x_{n}^{1}: n \in \mathbb{N}\right\}$. Then,

$$
\mathcal{V}=\mathcal{V}_{0} \cup \mathcal{V}_{1} \cup \mathcal{V}_{2} \text { and } \mathcal{E} \cap\left(\mathcal{V}_{0}^{2} \cup \mathcal{V}_{1}^{2} \cup \mathcal{V}_{2}^{2}\right)=\emptyset \text {. }
$$


So, $\mathcal{T}$ is tri-partite. Using Theorem 4.2, Proposition 7.3 and Proposition 7.12 , the result holds.

7.5. Triangular anti-tree. Let $\mathcal{T}=(\mathcal{V}, m, \mathcal{E}, \mathcal{R})$ be a weighted triangulation. The sphere of radius $n \in \mathbb{N}$ around a vertex $v \in \mathcal{V}$ is the set

$$
\mathcal{S}_{n}(v):=\left\{w \in \mathcal{V}: d_{\mathcal{V}}(v, w)=n\right\} .
$$

We recall that $\mathcal{C}_{n}$ denotes the $n$-cycle graph, i.e. $\mathcal{V}=\mathbb{Z} / n \mathbb{Z}$, where $\mathcal{E}(x, y)>0$ if and only if $|x-y|=1$. Let $\mathcal{G}\left(\mathcal{S}_{n}(o)\right)=\left(\mathcal{S}_{n}(v), \mathcal{E}^{\prime}\right)$ where $\mathcal{E}^{\prime}=\left.\mathcal{E}\right|_{\mathcal{S}_{n}(v) \times \mathcal{S}_{n}(v)}$.

Definition 7.14. Let $\mathcal{T}=(\mathcal{V}, m, \mathcal{E}, \mathcal{R})$ be a weighted triangulation. We say that $\mathcal{T}$ is anti-tree if there exists a vertex $o \in \mathcal{V}$ such that

1) For all $n \in \mathbb{N}^{*}$ and $v \in \mathcal{S}_{n}(o)$, we have

$$
\mathcal{N}_{\mathcal{G}}(v) \backslash \mathcal{S}_{n}(o)=\mathcal{S}_{n-1}(o) \cup \mathcal{S}_{n+1}(o) .
$$

2) For all $n \in \mathbb{N}^{*}, \mathcal{G}\left(\mathcal{S}_{n}(o)\right) \simeq \mathcal{C}_{\sharp \mathcal{S}_{n}(o)}$.

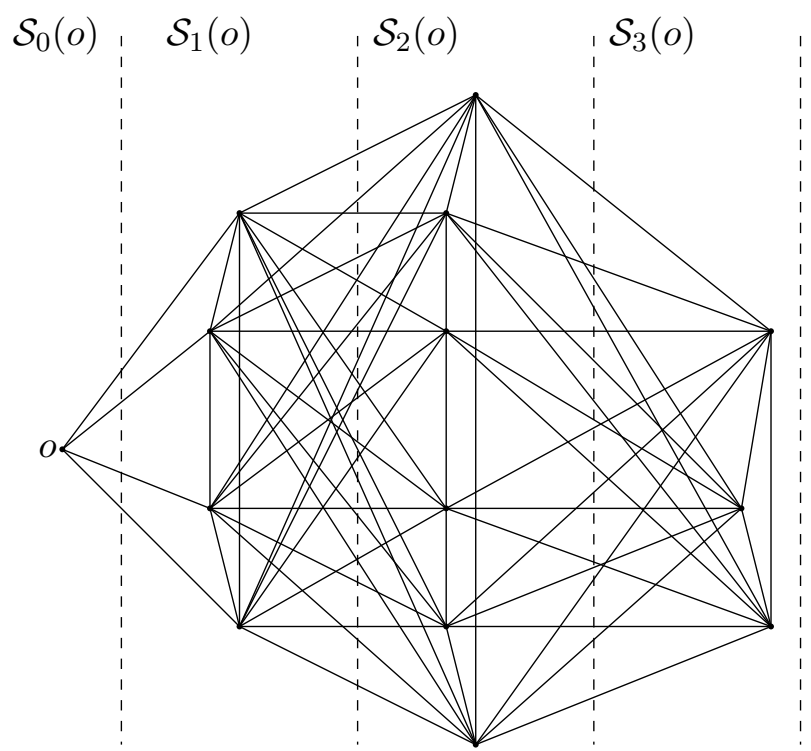

An triangular anti-tree with spheres $\mathcal{S}_{0}(o), \mathcal{S}_{1}(o), \mathcal{S}_{2}(o), \mathcal{S}_{3}(o)$.

Theorem 7.15. Let $\mathcal{T}=(\mathcal{V}, m, \mathcal{E}, \mathcal{R})$ be a simple triangular anti-tree whose root in o. Set $s_{n}=\sharp \mathcal{S}_{n}(o)$ and assume that

$$
n \longmapsto \frac{s_{n}^{2}}{s_{n+2}} \in \ell^{1}(\mathbb{N}) .
$$

Then $\mathcal{L}_{2, \mathrm{sym}}$ does not essentially self-adjoint on $\mathcal{C}_{\text {sym }}^{c}(\mathcal{R})$. 
Proof. Set $f \in \ell_{\text {sym }}^{2}(\mathcal{F}) \backslash\{0\}$ such that $f \in \operatorname{ker}\left(\mathcal{L}_{2, \text { sym }}^{*}+i\right)$ and such that $f$ is constant on $\mathcal{S}_{n} \times \mathcal{S}_{n+1}^{2} \cup \mathcal{S}_{n}^{2} \times \mathcal{S}_{n+1}, n \in \mathbb{N}^{*}$. We denote the constant value by $C_{n}$. It takes the value 0 on $\mathcal{S}_{n}^{2}$. We have the following equation:

$$
\left(s_{n}+4+i\right) C_{n}+s_{n+2} C_{n+1}=0 .
$$

Therefore,

$$
\begin{aligned}
\left\|\left.f\right|_{\mathcal{S}_{n+1} \times \mathcal{S}_{n+2}^{2}}\right\|^{2} & =\left\|\left.f\right|_{\mathcal{S}_{n+1}^{2} \times \mathcal{S}_{n+2}}\right\|^{2} \\
& =\frac{1}{2} s_{n+2} s_{n+1}\left|C_{n+1}\right|^{2} \\
& \leq \frac{\left|s_{n}+4+i\right|^{2}}{s_{n} s_{n+2}}\left\|\left.f\right|_{\mathcal{S}_{n} \times \mathcal{S}_{n+1}^{2}}\right\|^{2} .
\end{aligned}
$$

Since $\lim _{n \rightarrow \infty} \frac{s_{n}^{2}}{s_{n+2}}=0$, we get by induction:

$$
M:=\sup _{n \in \mathbb{N}^{*}}\left\|\left.f\right|_{\mathcal{S}_{n} \times \mathcal{S}_{n+1}^{2}}\right\|^{2}<\infty
$$

Then, we have

$$
\left\|\left.f\right|_{\mathcal{S}_{n+1} \times \mathcal{S}_{n+2}^{2}}\right\|^{2} \leq M \frac{\left|i+4+s_{n}\right|}{s_{n} s_{n+2}}
$$

From Eq. (3), we infer that $f \in \ell_{\text {sym }}^{2}(\mathcal{F})$. Using [26, Theorem X.36], we conclude that $\mathcal{L}_{2 \text {,sym }}$ is not essentially self-adjoint.

Corollary 7.16. Let $\mathcal{T}=(\mathcal{V}, m, \mathcal{E}, \mathcal{R})$ be a simple triangular anti-tree satisfying (3). Then, $\mathcal{A}_{\widehat{\mathcal{G}}}$ is not essentially self-adjoint on $\mathcal{C}^{c}(\widehat{\mathcal{V}})$.

\section{REFERENCES}

[1] Anné, C. and Torki-Hamza, N.: The Gauss-Bonnet operator of an infinite graph, Anal. Math. Phys., 5, No. 2, 137-159 (2015).

[2] Ayadi, H.: Spectra of Laplacians on an infinite graph, Oper. Matrices, 11, No. 2, 567-586 (2017).

[3] Baloudi, H., Golenia, S., and Jeribi, A.: The adjacency matrix and the discrete Laplacian acting on forms. arxiv preprint arxiv: 1505.06109(2015).

[4] Balti, M.: On the eigenvalues of weighted directed graphs, Complex Anal. Oper. Theory 11, No. 6, 1387-1406 (2017).

[5] Berkolaiko, G., Kennedy, J.B., Kurasov, P. and Mugnolo, D.: Edge connectivity and the spectral gap of combinatorial and quantum graphs, arXiv:1702.05264 [math.SP]

[6] Bonnefont, M. and Golénia, S.: Essential spectrum and Weyl asymptotics for discrete Laplacians, Ann. Fac. Sci. Toulouse Math. 24, No. 6, 563-624 (2015).

[7] Chebbi, Y.: The discrete Laplacian of a 2-Simplicial complex, Potential Anal., 49, No. 2, 331-358(2018)..

[8] Chebbi, Y.: Laplacien discret d'un 2-complexe simplicial, Doc. Thesis (PH.D), Universit de Nantes, Facult des sciences et des techniques; Universit de Carthage (Tunisie) (2018).

[9] Chung, F.R.K.: Spectral graph theory, Regional Conference Series in Mathematics. 92. Providence, RI: American Mathematical Society (AMS). xi, 1996. 
[10] Colin de Verdière, Y.: Spectres de graphes, Cours Spécialisés, 4. Société Mathématique de France, Paris, 1998.

[11] Colin de Verdière, Y., Torki-Hamza, N., Truc, F.: Essential self-adjointness for combinatorial schrödinger operators. II metrically non complete graphs, Math. Phys. Anal. Geom. 14, No. 1, 21-38 (2011).

[12] Cvetković, D., Doob, M., and Sachs, H.: Spectra of graphs. Theory and application, Second edition. VEB Deutscher Verlag der Wissenschaften, Berlin, 1982. $368 \mathrm{pp}$.

[13] Davidoff, G., Sarnak, P., and Valette, A.: Elementary number theory, group theory, and Ramanujan graphs, London Mathematical Society Student Texts, 55. Cambridge University Press, Cambridge, 2003. $\mathrm{x}+144$ pp.

[14] Doyle, P.G. and Snell, J.L.: Random walks and electric networks, The Carus Mathematical Monographs, the Mathematical Association of America, $159 \mathrm{pp}$. 1984.

[15] Golénia, S: Unboundedness of adjacency matrices of locally finite graphs, Lett. Math. Phys. 93, No. 2, 127-140 (2010).

[16] Golénia, S.: Hardy inequality and assymptotic eigenvalue distribution for discrete laplacians, J. Funct. Anal. 266, No. 5, 2662-2688 (2014).

[17] Golénia, S., and Schumacher, C.: The problem of deficiency indices for discrete Schrödinger operators on locally finite graph, J. Math. Phys. 52, No. 6, 063512, 17 p. (2011).

[18] Hung, X., Keller, M., Masamune, J., and Wojciechowski, R.K: A note on selfadjoint extensions of the Laplacian on weighted graphs, J. Funct. Anal. 265, No. 8, 1556-1578 (2013).

[19] Jeribi, A.: Spectral theory and applications of linear operators and block operator matrices. New York (NY): Springer-Verlag, 2015.

[20] Kato, T.: Perturbation theory for linear operators. New York (NY): springerVerlag, 1966.

[21] Keller, M.: The essential spectrum of the Laplacian on rapidly branching tessellations, Math. Ann. 346, No. 1, 51-66 (2010).

[22] Milatovic, O.: Essential self-adjointness of magnetic Schrödinger operators on locally finite graphs, Integral Equations Oper. Theory 71, No. 1, 13-27 (2011).

[23] Mohar, B. and Omladič M.: The spectrum of infinite graphs with bounded vertex degrees, Graphs, hypergraphsGraphs, hypergraphs and applications, Proc. Conf. Graph Theory, Eyba/GDR 1984, Teubner-Texte Math. 73, 122-125 (1985).

[24] Mohar, B. and Woess, W.: A survey on spectra of infinite graphs, J. Bull. Lond. Math. Soc. 21, No.3, 209-234 (1989).

[25] Palle E. T. Jørgensen: Essential self-adjointness of semibounded operators. Math. Ann. 237, No. 2, 187-192 (1978).

[26] Reed, M. and Simon, B.: Methods of modern mathematical physics tome I-IV, Academic Press 1978.

[27] Schechter, M.: Principles of functional analysis. Academic Press, 1971.

[28] Shirai, T.: The spectrum of infinite regular line graphs. Trans. Am. Math. Soc. 352, No. 1, 115-132 (2000).

[29] Weber, A.: Analysis of the physical Laplacian and the heat flow on a locally finite graph, J. Math. Anal. Appl. 370, No. 1, 146-158 (2010).

[30] Wolf, F.: On the essential spectrum of partial differential boundary problems, Comm. Pure Appl. Math. 12, No. 2, 211-228 (1959). 
Hatem Baloudi, Department of Mathematics, Faculty of Sciences of Gafsa, University of Gafsa, 2112 Zarroug, Tunisia

Sayda Belgacem Aref Jeribi, Department of Mathematics, Faculty of SciEnCES of Sfax, University of Sfax, 3000 Sfax, Tunisia 